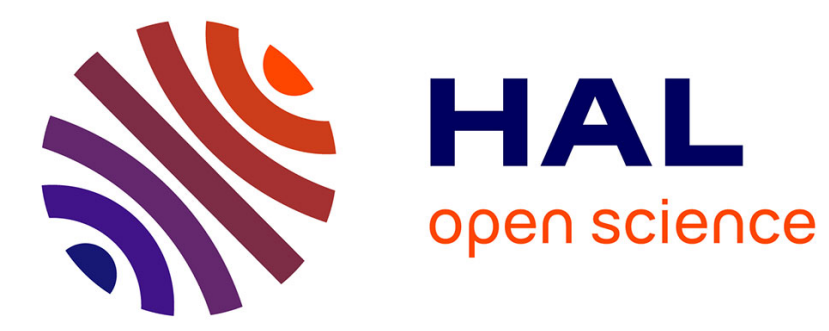

\title{
A simple proof that generic 3-RPR manipulators have two aspects \\ Michel Coste
}

\section{To cite this version:}

Michel Coste. A simple proof that generic 3-RPR manipulators have two aspects. Journal of Mechanisms and Robotics, 2012, 4 (1), 10.1115/1.4005333 . hal-00468453v2

\section{HAL Id: hal-00468453 \\ https://hal.science/hal-00468453v2}

Submitted on 15 Nov 2011

HAL is a multi-disciplinary open access archive for the deposit and dissemination of scientific research documents, whether they are published or not. The documents may come from teaching and research institutions in France or abroad, or from public or private research centers.
L'archive ouverte pluridisciplinaire HAL, est destinée au dépôt et à la diffusion de documents scientifiques de niveau recherche, publiés ou non, émanant des établissements d'enseignement et de recherche français ou étrangers, des laboratoires publics ou privés. 


\title{
A Simple Proof that Generic 3-RPR Manipulators Have Two Aspects
}

\author{
Michel Coste \\ Institut de Recherche Mathématique de Rennes (IRMAR) \\ CNRS : UMR 6625 - Université de Rennes I, France \\ E-mail: michel.coste@univ-rennes1.fr
}

\begin{abstract}
Avoiding singularities in the workspace of a parallel robot is an important issue. The case of 3-RPR planar robots is an important subject of theoretical studies. We study the singularities of planar 3-RPR robots by using a new parameterization of the singular locus in a modified workspace. This approach enables us to give a simple alternative proof of a result recently proved by M. Husty: the complement of the singular locus in the workspace of a generic 3-RPR manipulator has two connected components (called aspects). The parameterization introduced in this paper, due to its simple geometric properties, proves to be useful for the study of the singularities of 3-RPR robots.
\end{abstract}

Keywords: parallel robots, singularities

\section{Introduction}

Planar 3-RPR manipulators have been extensively studied because they meet several interesting features such as potential industrial applications, relative kinematic simplicity and nice mathematical properties [1-12]. Moreover, the study of the 3RPR planar manipulator may help to better understand the kinematic behaviour of its more complex spatial counterpart, the 6-d.o.f. octahedral manipulator, as reported in [3]. An important feature of these manipulators is their ability to change assembly mode without encountering a singularity [1-6]. Since a parallel manipulator becomes uncontrollable on a singular (for the direct kinematic problem) configuration, this feature is interesting as it can enlarge its usable workspace. Knowing whether a parallel manipulator, or, more interestingly, a family of manipulators has this feature or not is of interest for both the designer and the end-user. Determining the number of aspects (singularity-free domain) may help answering this question.

A well-known conjecture in the kinematics community was that a generic 3-RPR manipulator has two aspects. Recently [6], M. Husty published a proof of this conjecture. Since, in the same time, there are up to 6 assembly modes, this shows that a generic manipulator has more than one assembly mode in one of its aspects, thus showing that non-singular assembly mode changing motions are possible. The proof by M. Husty is rather technical and involved, and the details of the proof are yet to appear in a joint paper with J. Schicho. We propose here a much simpler proof which is based on a parameterization of the singular surface different from the one used by M. Husty and on arguments of a simple topological nature.

The rest of this paper is organized as follows. Section 2 describes a modified workspace and the new parameterization of the singular surface in this modified workspace. Section 3 introduces useful tools which are the curves of zeros and poles of this parameterization. Section 4 is devoted to the proof that there are generically two aspects, including a method to design a; path between points in the same aspect. We also discuss in this section the non-generic cases and the distribution of the solutions of the direct kinematic problem in the two aspects.

\section{The parameterization of the singular surface}

\subsection{Notations}

In order to describe the manipulator, we use here the following notations (see Fig. 1): 
The base triangle is $A_{1} A_{2} A_{3}$ (with the direct orientation). We take $b_{A}=A_{1} A_{2}$ as the base of this triangle; the coordinates of the point $A_{3}$ in the direct orthonormal frame $\mathcal{F}$ with origin $A_{1}$ and first coordinate axis directed and oriented by $\overrightarrow{A_{1} A_{2}}$ are denoted by $\left(d_{A}, h_{A}\right)$.

The moving triangle is denoted by $B_{1} B_{2} B_{3}$ where $B_{i}$ is linked to $A_{i}$ by a leg of the manipulator $\left(B_{1} B_{2} B_{3}\right.$ may be in indirect orientation). We use the parameters $\left(b_{B}, h_{B}, d_{B}\right)$, analogous to the ones defined above for the base triangle, to encode the geometry of the moving triangle. Note that $h_{B}$ may be negative.

We assume that neither the base nor the moving triangles are flat, i.e. none of $b_{A}, h_{A}, b_{B}$ and $h_{B}$ is 0 .

\subsection{The modified workspace}

The workspace $W$ of the manipulator is the space of planar rigid motions. Usually, the position of the moving triangle with respect to the base triangle is given by the coordinates $(\varphi, x, y)$ where

the angular coordinate $\varphi$ measures the angle $\left(\overrightarrow{A_{1} A_{2}}, \overrightarrow{B_{1} B_{2}}\right)$ modulo $2 \pi$, the coordinates $(x, y)$ are the coordinates of $B_{1}$ in the frame $\mathcal{F}$ defined above.

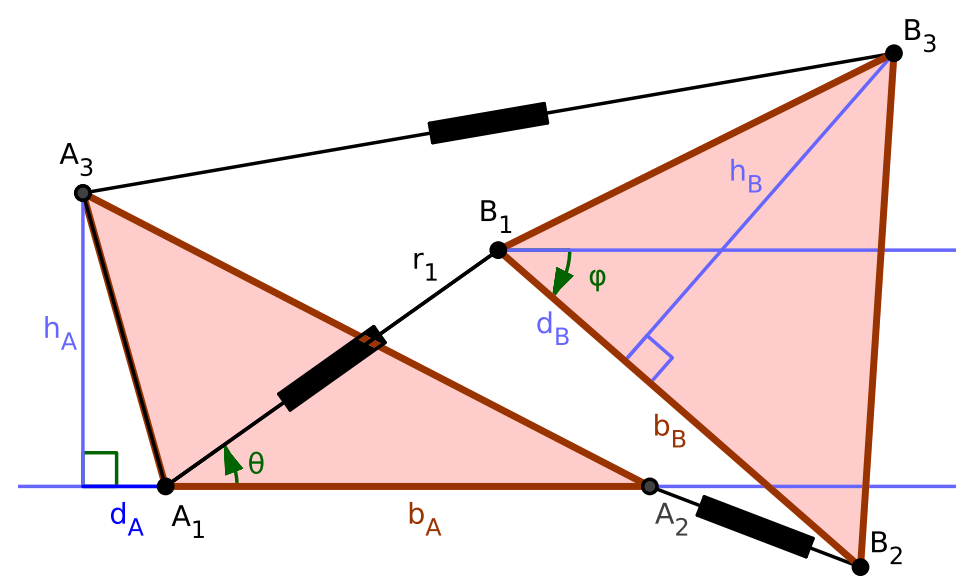

Fig. 1. Parameters and coordinates

We propose here to use a modified workspace $\widetilde{W}$ where the coordinates of the translation part of the rigid motion are some kind of polar coordinates. More precisely, $\widetilde{W}$ is the parameter space for a parameterization $m: \widetilde{W} \rightarrow W-$ not everywhere one-to-one - of the workspace, but we shall call it "modified workspace" for short. The modification $m$ is defined by $m\left(\varphi, \theta, r_{1}\right)=\left(\varphi, r_{1} \cos (\theta), r_{1} \sin (\theta)\right)$ where

$\varphi$ is the same as above,

$\theta \in[-\pi / 2, \pi / 2]$ and $r_{1} \in \mathbb{R}$,

modulo the identification of $\left(\varphi, \pi / 2, r_{1}\right)$ with $\left(\varphi,-\pi / 2,-r_{1}\right)$ (see Fig. 1$)$.

Note that $r_{1}$ may be negative; its absolute value is the length of the first leg of the manipulator. The angle $\theta$ is an oriented angle of lines (the angle of lines $\left(A_{1} A_{2}\right)$ and $\left(A_{1} B_{1}\right)$, if $B_{1}$ is distinct from $\left.A_{1}\right)$ and it is measured modulo $\pi$.

It is in order here to comment the use of this modified workspace $\widetilde{W}$. The coordinates we shall use have the peculiarity that all triples $(\varphi, \theta, 0)$ with $\varphi$ fixed and $\theta$ varying from $-\pi / 2$ to $\pi / 2$ correspond via $m$ to the same position of the manipulator. In some sense, we choose arbitrarily a direction for the first leg of the manipulator, although its length is null. From the algebro-geometric point of view, we have blown up the origin $0 \in \mathbb{R}^{2}$, getting thus the Moebius strip $[-\pi / 2, \pi / 2] \times \mathbb{R}$ where $\left(\pi / 2, r_{1}\right)$ is identified with $\left(-\pi / 2,-r_{1}\right)$. The use of these coordinates may look awkward at first sight, but it will enable us to obtain a useful parameterization of the singular surface in our modified workspace, with $r_{1}$ as a function of $\varphi$ and $\theta$.

The modification $m: \widetilde{W} \rightarrow W$ is one-to-one outside of the set $x=y=0$ in $W$. This set is contained in the singular set (the configurations with $B_{1}=A_{1}$ are singular). The set $x=y=0$ is a circle in $W$, parameterized by $\varphi$; its inverse image by $m$ in $\widetilde{W}$ is the torus $r_{1}=0$, parameterized by $(\varphi, \theta)$. Hence, the singular set is changed when it is pulled back along $m: \widetilde{W} \rightarrow W$. Actually, this change of the singular set makes easier to understand its geometry. However, we are primarily interested in the 
complement of the singular set in the workspace, and this complement is not affected by pulling back along $m$. Hence, the number of aspects can well be determined by using $\widetilde{W}$.

We denote by $T$ the torus $\mathbb{R} / 2 \pi \mathbb{Z} \times \mathbb{R} / \pi \mathbb{Z}$ with coordinates $(\varphi, \theta)$. We denote by $p: \widetilde{W} \rightarrow T$ the projection defined by $p\left(\varphi, \theta, r_{1}\right)=(\varphi, \theta)$. Note that, for each point $(\varphi, \theta)$ of the torus $T$, the fiber $p^{-1}(\varphi, \theta)$ of the projection is a line, but $\widetilde{W}$ is not globally homeomorphic to the product $T \times \mathbb{R}$; the modified workspace $\widetilde{W}$ is actually a nontrivial line bundle over $T$.

\subsection{Parameterization of the singular surface}

The equation of the singular locus in the modified workspace $\widetilde{W}$ is obtained by expressing the fact that the legs $\left(A_{1} B_{1}\right)$, $\left(A_{2} B_{2}\right)$ and $\left(A_{3} B_{3}\right)$ are concurrent or parallel; this is done by equating a $3 \times 3$ determinant to 0 . There is of course a factor $r_{1}$ in this equation (a result of the blowing up of the origin of $\mathbb{R}^{2}$ ). The other factor (which is actually the equation of the strict transform of the singular surface by the blowing up) has the form

$$
N(\varphi, \theta)-D(\varphi, \theta) r_{1}
$$

where $N(\varphi, \theta)$ and $D(\varphi, \theta)$ are polynomials in the trigonometric functions of $\varphi$ and $\theta$. Precisely, we have:

$$
\begin{aligned}
N(\varphi, \theta)= & b_{B}\left(\left(b_{A} d_{B}-h_{A} h_{B}-d_{A} d_{B}\right)(\sin \varphi)^{2}-b_{A} h_{A} \sin \varphi\right. \\
& \left.+\left(h_{A} d_{B}+b_{A} h_{B}-d_{A} h_{B}\right) \sin \varphi \cos \varphi\right) \cos \theta \\
& +\left(b_{A}\left(h_{A} d_{B}-d_{A} h_{B}\right) \cos \varphi+b_{A}\left(d_{A} b_{B}-d_{A} d_{B}-h_{A} h_{B}\right) \sin \varphi\right. \\
& +b_{A} b_{B} h_{B}+b_{B}\left(d_{A} h_{B}-b_{A} h_{B}-h_{A} d_{B}\right)(\cos \varphi)^{2} \\
& \left.+b_{B}\left(h_{A} h_{B}+d_{A} d_{B}-b_{A} d_{B}\right) \cos \varphi \sin \varphi\right) \sin \theta
\end{aligned}
$$

and

$$
\begin{aligned}
D(\varphi, \theta)= & \left(\left(b_{A} d_{B}-d_{A} b_{B}\right)(\sin \theta)^{2}+\left(h_{A} b_{B}-b_{A} h_{B}\right) \cos \theta \sin \theta\right) \cos \varphi \\
& +\left(\left(d_{A} b_{B}-b_{A} d_{B}\right) \sin \theta \cos \theta-h_{A} b_{B}(\cos \theta)^{2}-b_{A} h_{B}(\sin \theta)^{2}\right) \sin \varphi .
\end{aligned}
$$

In the following, we shall call singular surface the surface in $\widetilde{W}$ with equation $N(\varphi, \theta)-D(\varphi, \theta) r_{1}=0$ and singular locus the union of this surface with $r_{1}=0$.

Solving Eq. (1) in $r_{1}$ we get the parameterization $r_{1}^{\text {Sing }}(\varphi, \theta)=\frac{N(\varphi, \theta)}{D(\varphi, \theta)}$. Observe that we have $r_{1}^{\text {Sing }}(\varphi, \theta+\pi)=$ $-r_{1}^{\text {Sing }}(\varphi, \theta)$, which agrees with the identification made in the description of the modified workspace.

From an algebro-geometric point of view, the singular locus in the line bundle $\widetilde{W}$ consists of two sections of this line bundle over $T$ : the zero section $r_{1}=0$ and a rational section given by $r_{1}^{\text {Sing }}(\varphi, \theta)$. This relatively simple description will allow us to have a good control on the topology of the complement.

The parameterization $r_{1}^{\text {Sing }}(\varphi, \theta)$ gives an infinite value for $r_{1}^{\text {Sing }}$ when $D(\varphi, \theta)=0$, and the zero value when $N(\varphi, \theta)=0$. These two equations describe curves on the torus $T$ which is the space of the angular coordinates $(\varphi \bmod 2 \pi, \theta \bmod \pi)$. The curve $D(\varphi, \theta)=0$ will be called the curve of poles of the parameterization, and the curve $N(\varphi, \theta)=0$ its curve of zeros. The points of intersection of the two curves are the indetermination points of the parameterization $r_{1}^{\text {Sing }}(\varphi, \theta)$. These two curves will play a prominent role in the determination of the connected components of the complement of the singular locus.

\section{Poles and zeros of the parameterization}

\subsection{Example}

We consider the manipulator with parameters $b_{A}=15.9, h_{A}=10, d_{A}=0, b_{B}=17, h_{B}=16.1, d_{B}=13.2$. These parameters correspond to the Innocenti-Merlet manipulator [14]. Figure 2 shows the curve of zeros $N(\varphi, \theta)=0$ in dashed line, and the curve of poles $D(\varphi, \theta)=0$ in solid line. The two curves intersect in eight indetermination points indicated by diamonds. 


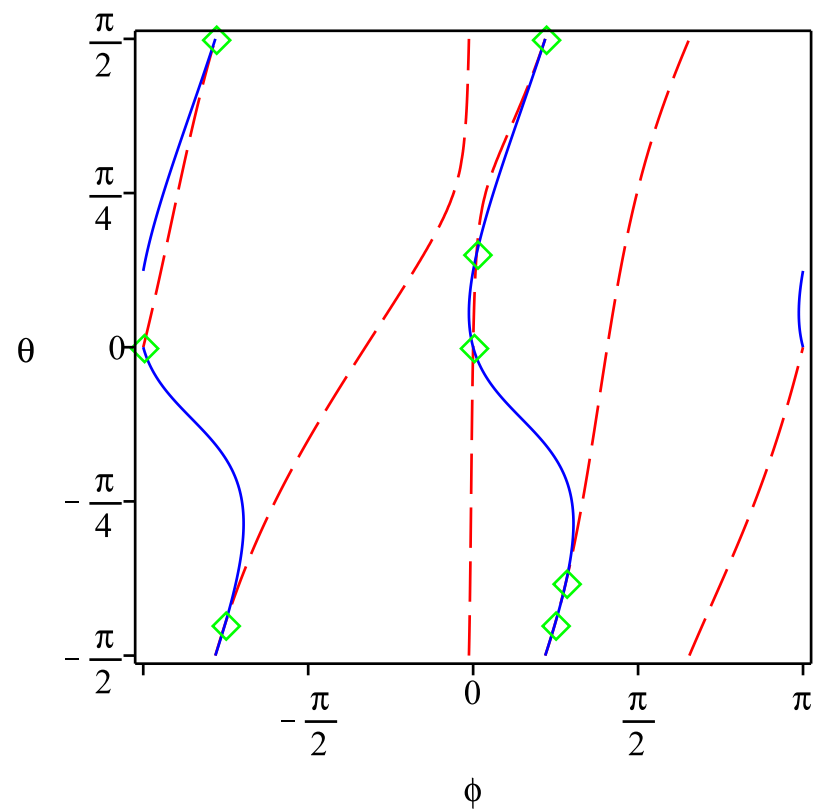

Fig. 2. Curves of poles and zeros for the Innocenti-Merlet manipulator

Recall that the space of $(\varphi, \theta)$ is actually a torus $T$ obtained by gluing the left side of the picture with the right side, and the bottom side with the top side. Taking into account these identifications, we see that the curve of poles has two connected components (two branches), visibly corresponding one to the other in the translation $\varphi \mapsto \varphi+\pi \quad(\bmod 2 \pi)$, while the curve of zeros has only one connected component. The two branches of the curve of poles cut the torus $T$ in two cylinders. The complement of the union of the curves in $T$ has eight connected components. Six among them are rather small and some are not even easily distinguished in Fig. 2.

\subsection{The curve of poles}

The formula (3) for $D(\varphi, \theta)$ shows that $D(\varphi, \theta)=0$ can be solved in $\tan \varphi$. Specifically, we have

$$
\tan (\varphi)=\frac{\left(h_{A} b_{B}-b_{A} h_{B}\right) \sin (\theta) \cos (\theta)+\left(b_{A} d_{B}-d_{A} b_{B}\right) \sin (\theta)^{2}}{h_{A} b_{B} \cos (\theta)^{2}+\left(b_{A} d_{B}-d_{A} b_{B}\right) \cos (\theta) \sin (\theta)+b_{A} h_{B} \sin (\theta)^{2}}
$$

This formula gives, for each value of $\theta$, two values of $\varphi$ modulo $2 \pi$ which differ by $\pi$. This is rather clear from a geometric point of view, since the "asymptotic" singular situations (i.e., those with $r_{1}^{\text {Sing }}$ infinite) are characterized by the fact that

$$
\frac{\overline{\ell\left(A_{1}\right) \ell\left(A_{2}\right)}}{\overline{\ell\left(A_{1}\right) \ell\left(A_{3}\right)}}=\frac{\overline{\ell\left(B_{1}\right) \ell\left(B_{2}\right)}}{\overline{\ell\left(B_{1}\right) \ell\left(B_{3}\right)}},
$$

where $\ell$ is a projection parallel to the common direction of the infinite legs, which is given by $\theta$; this is realized by two orientations of the moving triangle with respect to the base (encoded by the angle $\varphi$ ) which differ by $\pi$.

Note that the curve of poles has no "horizontal" component, i.e. no component of the form $\theta=$ constant. This could happen only if the numerator and denominator in (4) would both vanish for some value of $\theta$. It can be easily checked that this is never the case, whatever values are given to $b_{A}, h_{A}, d_{A}, b_{B}, h_{B}, d_{B}$ (always assuming neither triangle is flat).

We have seen in the example that the curve of poles has two branches (or connected components) one of which is obtained from the other by the translation $\varphi \mapsto \varphi+\pi$. This is always the case. This point, which will be of importance later, is not a priori clear, since there could be only one branch possessing the translation symmetry $\varphi \mapsto \varphi+\pi$. But indeed the equation $\cos \varphi=0$, together with $D(\varphi, \theta)=0$, is an equation of degree 2 in $\tan \theta$, precisely

$$
b_{A} h_{B}(\tan \theta)^{2}+\left(b_{A} d_{B}-b_{B} d_{A}\right) \tan \theta+b_{B} h_{A}=0
$$


This equation has 0 or 2 solutions in $\theta$. The fact that this number is even implies that, if one follows continuously a determination of $\varphi$ along $D(\varphi, \theta)=0$ when $\theta$ varies from $-\pi / 2$ to $\pi / 2$, one returns to the same determination of $\varphi$ modulo $2 \pi$. Hence, there are two disjoint branches of the curve $D(\varphi, \theta)=0$. One of the branch intersects $\theta=0$ in $(0,0)$ and the other in $(\pi, 0)=(-\pi, 0)$.

The two branches of the curve $D(\varphi, \theta)=0$ cut the torus $T$ in two cylinders which are image one of the other by $\varphi \mapsto \varphi+\pi$ $(\bmod 2 \pi)$. Denote by $T_{1}$ the cylinder containing $(-\pi / 2,0)$ and by $T_{2}$ the one containing $(\pi / 2,0)$.

\subsection{The curve of zeros}

Considering the formula (2) for $N(\varphi, \theta)$, it appears that $N(\varphi, \theta)=0$ can be solved in $\tan (\theta)$. Hence, we obtain a function $v: \varphi \bmod 2 \pi \mapsto \theta \bmod \pi$. This can be explained from a geometric point of view : the angle $\theta$ is the angle from the line $\left(A_{1} A_{2}\right)$ to the line joining $A_{1}$ to the intersection point of the legs $\left(A_{2} B_{2}\right)$ and $\left(A_{3} B_{3}\right)$, when $B_{1}=A_{1}$ and the angle $\left(\overrightarrow{A_{1} A_{2}}, \overrightarrow{B_{1} B_{2}}\right)$ is equal to $\varphi$.

It may be the case that the curve of zeros is not only the graph of the function $v$, but has also components which are "vertical" lines $\varphi=$ constant. These special values of $\varphi$ are those for which the coefficients of $\cos \theta$ and $\sin \theta$ in (2) both vanish. We shall return to these non generic cases in section 4.3.

\subsection{Indetermination points}

For a generic 3-RPR manipulator, the two curves $N(\varphi, \theta)=0$ and $D(\varphi, \theta)=0$ on the torus $T$ have no common component and intersect in finitely many points which are the indetermination points of the parameterization.

The indetermination points can be computed using the resultant of $N(\varphi, \theta)$ and $D(\varphi, \theta)$ with respect to $\tan \theta$. This resultant has four factors

$$
\begin{aligned}
F_{1}(\varphi)= & b_{A} b_{B} \sin \varphi \\
F_{2}(\varphi)= & \left(d_{A} h_{B}-h_{A} d_{B}\right) \cos \varphi+\left(h_{A} h_{B}+d_{A} d_{B}\right) \sin \varphi \\
= & A_{1} A_{3} \times B_{1} B_{3} \times \sin \left(\varphi-\alpha_{1}+\beta_{1}\right) \\
F_{3}(\varphi)= & \left(d_{A} h_{B}-h_{A} d_{B}+b_{B} h_{A}-b_{A} h_{B}\right) \cos \varphi \\
& \quad+\left(d_{A} d_{B}+b_{A} b_{B}+h_{A} h_{B}-b_{B} d_{A}-b_{A} d_{B}\right) \sin \varphi \\
= & A_{2} A_{3} \times B_{2} B_{3} \times \sin \left(\varphi+\alpha_{2}-\beta_{2}\right) \\
F_{4}(\varphi)= & \left(-b_{A} h_{B}-b_{B} h_{A}\right) \cos \varphi+\left(-b_{A} d_{B}+b_{B} d_{A}\right) \sin \varphi+b_{A} h_{A}+b_{B} h_{B}
\end{aligned}
$$

where we use the angles $\alpha_{1}=\left(\overrightarrow{A_{1} A_{2}}, \overrightarrow{A_{1} A_{3}}\right), \beta_{1}=\left(\overrightarrow{B_{1} B_{2}}, \overrightarrow{B_{1} B_{3}}\right), \alpha_{2}=\left(\overrightarrow{A_{2} A_{3}}, \overrightarrow{A_{2} A_{1}}\right)$ and $\beta_{2}=\left(\overrightarrow{B_{2} B_{3}}, \overrightarrow{B_{2} B_{1}}\right)$.

The vanishing of the first three factors corresponds to the parallelism of the sides $\left[A_{1} A_{2}\right]$ and $\left[B_{1} B_{2}\right]$ (respectively $\left[A_{1} A_{3}\right]$ and $\left[B_{1} B_{3}\right],\left[A_{2} A_{3}\right]$ and $\left.\left[B_{2} B_{3}\right]\right)$. The fact that this gives rise to situations where the singular value of $r_{1}$ is not determined has an easy geometric interpretation: this is for instance the case if the two sides $\left[A_{1} A_{2}\right]$ and $\left[B_{1} B_{2}\right]$ are on the same line.

There is clearly another situation where the singular value of $r_{1}$ is not determined: when the three legs of the manipulator are parallel. The conditions for the parallelism of the second and third legs with the first one are as follows:

$$
\begin{aligned}
b_{A} \sin \theta & =b_{B} \sin (\theta-\varphi) \\
d_{A} \sin \theta-h_{A} \cos \theta & =d_{B} \sin (\theta-\varphi)-h_{B} \cos (\theta-\varphi) .
\end{aligned}
$$

Writing these equation in $\tan \theta$ and taking the resultant with respect to $\tan \theta$ gives indeed the fourth factor, up to sign.

Each of these first three factors has two solutions in $\varphi$ modulo $2 \pi$ which differ by $\pi$. The last factor may have or not two solutions in $\varphi$. Hence, for a generic 3-RPR manipulator, the parameterization has 6 or 8 indetermination points, possibly counted with multiplicity. The non generic case is the case when the resultant of $N(\varphi, \theta)$ and $D(\varphi, \theta)$ with respect to tan $\theta$ is identically zero. We shall return to this case in section 4.3.

\section{Proof that there are generically two aspects}

We now proceed to prove that a generic 3-RPR manipulator has two aspects, i.e. that the complement of the singular locus in $\widetilde{W}$ has two connected components. Recall that this singular locus is the union of $r_{1}=0$ and of $N(\varphi, \theta)-r_{1} D(\varphi, \theta)=$ 0 . Recall also that the curve of poles $D(\varphi, \theta)=0$ in the torus $T$ has two branches which cut $T$ in two cylinders $T_{1}$ and $T_{2}$. 


\subsection{The unbounded components above the cylinders $T_{i}$}

Let us examine what happens in the part $p^{-1}\left(T_{i}\right)$ of the modified workspace $\widetilde{W}$ which is above the cylinder $T_{i}$. The section $r_{1}^{\text {Sing }}(\varphi, \theta)$ is defined over $T_{i}$ since the denominator $D(\varphi, \theta)$ does not vanish on $T_{i}$, and the singular locus in $p^{-1}\left(T_{i}\right)$ is the union of the two sections

$$
\begin{aligned}
& (\varphi, \theta) \longmapsto r_{1}=0 \\
& (\varphi, \theta) \longmapsto r_{1}=r_{1}^{\text {Sing }}(\varphi, \theta)
\end{aligned}
$$

over $T_{i}$. Locally, these two sections can be viewed as continuous functions with values in $\mathbb{R}$ and, in the complement of their graphs, there is a connected part which is "above" both and another connected part which is "under" both. Now recall that we have the identification of $\left(\varphi, \theta, r_{1}\right)$ with $\left(\varphi, \theta+\pi,-r_{1}\right)$. Hence, when one makes one turn on the cylinder $T_{i}$ going from $\theta=-\pi / 2$ to $\theta=\pi / 2$, the part of the complement of the singular locus over $T_{i}$ which is "above" both sections is glued with the part which is "under" both sections. In conclusion, there is only one unbounded connected component of the complement of the singular locus in $p^{-1}\left(T_{i}\right)$.

Given any two points belonging to the unbounded component in $p^{-1}\left(T_{i}\right)$, there is a continuous path joining them inside this component. We give an explicit procedure to describe such a path for two points in $p^{-1}\left(T_{2}\right)$. Denote by $\varphi=\tau(\theta)$ the parameterization of the branch of the curve of poles passing through $(0,0)$, so that $T_{2}$ is the cylinder of $(\varphi, \theta)$ such that $0<\varphi-\tau(\theta)<\pi \quad(\bmod 2 \pi)$. It will be convenient to allow $\theta$ to vary from $-\pi / 2$ to $3 \pi / 2$, always identifying $\left(\varphi, \theta+\pi, r_{1}\right)$ with $\left(\varphi, \theta,-r_{1}\right)$; then one can describe the unbounded component in $p^{-1}\left(T_{2}\right)$ as the set of $\left(\varphi, \theta, r_{1}\right)$ with $-\pi / 2 \leq \theta<3 \pi / 2$, $0<\varphi-\tau(\theta)<\pi(\bmod 2 \pi)$ and $r_{1}>\max \left(0, r_{1}^{\text {Sing }}(\varphi, \theta)\right)$. Hence, the unbounded component is the image of $(0, \pi) \times$ $[-\pi / 2,3 \pi / 2) \times(0,+\infty)$ by the continuous mapping

$$
(\eta, \theta, s) \longmapsto\left(\tau(\theta)+\eta, \theta, \max \left(0, r_{1}^{\text {Sing }}(\tau(\theta)+\eta, \theta)\right)+s\right)
$$

We can take, as path joining two points in the unbounded component, the image by the mapping above of the segment joining the two corresponding points in $(0, \pi) \times[-\pi / 2,3 \pi / 3) \times(0,+\infty)$.

It remains to understand the bounded connected components (those which are comprised between the two sections), and how these components are glued together when passing from one cylinder to the other.

\subsection{The bounded components}

Each cylinder $T_{i}$ is cut into finitely many connected components by the curve of zeros. The sections $r_{1}=0$ and $r_{1}=$ $r_{1}^{\text {Sing }}(\varphi, \theta)$ are defined, continuous and nowhere equal on each of these connected components $C$. Hence, the complement of the singular locus in $p^{-1}(C) \subset \widetilde{W}$ has one bounded connected component comprised between the two sections.

We make now the following genericity assumptions.

1. The curves $N(\varphi, \theta)=0$ and $D(\varphi, \theta)=0$ have no common component.

2. The curve $N(\varphi, \theta)=0$ has no "vertical line" ( $\varphi=$ constant) component.

We consider the consequences of the first genericity assumption. The curves $N(\varphi, \theta)=0$ and $D(\varphi, \theta)=0$ have only finitely many indetermination points in common. When one crosses one branch of the curve $D(\varphi, \theta)=0$ outside of these indetermination points, passing from the cylinder $T_{1}$ to $T_{2}$ or vice-versa, then $D(\varphi, \theta)=0$ changes sign while $N(\varphi, \theta)$ keeps its sign. Hence, $r_{1}^{\text {Sing }}(\varphi, \theta)$ jumps from $-\infty$ to $+\infty$ or vice-versa. Hence, the unbounded component of the complement of the singular locus over $T_{1}$ is glued with a bounded component comprised between the sections $r_{1}=0$ and $r_{1}=r_{1}^{\text {Sing }}(\varphi, \theta)$ over $T_{2}$, and vice-versa (see Fig. 3).

We turn now to the consequences of the second genericity assumption. It implies that the curve of zeros is consisting only of the graph of the function $v: \varphi \bmod 2 \pi \mapsto \theta \bmod \pi$. So it is topologically a circle contained in the torus $T$ and this circle cannot bound (i.e., it is not homologically trivial). Now consider a connected component $C$ cut by the curve of zeros in the cylinder $T_{i}$. This connected component has in its boudary at least one segment of a branch of the curve of poles. Indeed, otherwise $C$ would have as its boundary the whole curve of zeros, by Jordan's curve theorem; this is in contradiction with the fact that the curve of zeros is not homologically trivial.

We can give an alternative argument to show that $C$ cannot be bounded only by the curve of zeros, but must have at least one segment of a branch of the curve of poles in its boundary. If one makes a turn on the torus, with $\theta$ going from $-\pi / 2$ to $\pi / 2$, following a branch of the curve of poles, say on its left side, then one has to cross the curve of zeros an odd number of times since $r_{1}^{\text {Sing }}(\varphi, \pi / 2)=-r_{1}^{\text {Sing }}(\varphi,-\pi / 2)$. Hence, there are an odd number of indetermination points, possibly counted 


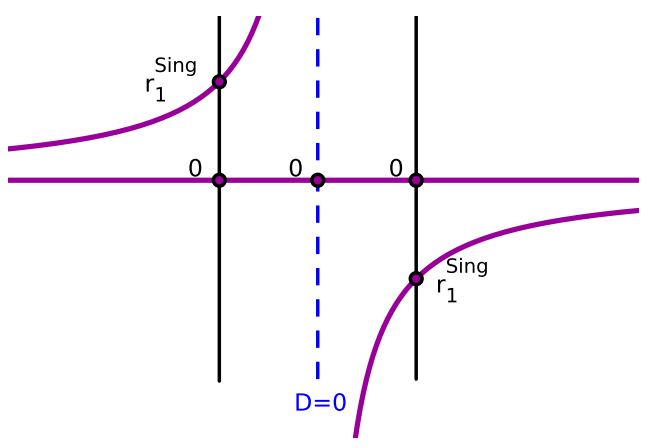

Fig. 3. Crossing the curve of poles

with multiplicity, on each branch of the curve of poles (precisely, 3 or 5 following the discussion in section 3.4). This implies that the curve of zeros has to cross each branch of the curve of poles.

In conclusion, each bounded connected component of the complement of the singular locus over a cylinder $T_{i}$ is glued with the unbounded component over the other cylinder through a segment of a branch of the curve of poles. We completed the proof of the result of M. Husty:

\section{Theorem 1. A generic planar 3-RPR manipulator has two aspects.}

We supplement the proof with the description of a path joining a point in a bounded component in $p^{-1}\left(T_{1}\right)$ to the unbounded component in $p^{-1}\left(T_{2}\right)$; combining this with what has been done for the unbounded component, we can design a path between any two points in the same aspect.

Assume the starting point is $\left(\varphi_{0}, \theta_{0}, r_{1,0}\right)$ with $0<r_{1,0}<r_{1}^{\text {Sing }}\left(\varphi_{0}, \theta_{0}\right)$ (the case $0>r_{1,0}>r_{1}^{\text {Sing }}\left(\varphi_{0}, \theta_{0}\right)$ is dealt with in a similar way). The graph of $\varphi \mapsto \mu(\varphi)=\theta_{0}+v(\varphi)-v\left(\varphi_{0}\right)$ is parallel to the curve of zeros and crosses the curve of poles for some $\varphi_{1}$, entering the cylinder $T_{2}$. The segment of the graph of $\mu$ between $\varphi_{0}$ and $\varphi_{1}$ is lifted to a continuous path in the bounded component using

$$
\varphi \mapsto\left(\varphi, \mu(\varphi), r_{1,0} \min \left(1, r_{1}^{\mathrm{Sing}}(\varphi, \mu(\varphi)) / r_{1}^{\mathrm{Sing}}\left(\varphi_{0}, \theta_{0}\right)\right)\right)
$$

where we take a continuous determination of $\mu \bmod \pi$ so that $r_{1}^{\mathrm{Sing}}(\varphi, \mu(\varphi))$ is positive.

\subsection{Non-generic cases}

We now discuss briefly the non-generic cases. Recall that we only consider manipulators where neither the base triangle nor the moving triangle are flat (i.e. $b_{A} h_{A} b_{B} h_{B} \neq 0$ ).

The most severe failure to genericity is the case when the two curves $N(\varphi, \theta)=0$ and $D(\varphi, \theta)=0$ have a common component (i.e., our genericity assumption 1 is not fulfilled). The restriction of the line bundle to this common component is entirely contained in the singular locus, and this kind of "wall" forbides gluing connected pieces of the complement of the singular locus. This case can be determined by computing resultants, and it occurs precisely for the following peculiar geometries of the manipulator:

1. "Similar" manipulators where the moving triangle and the base triangle are directly similar (i.e $b_{B}=\lambda b_{A}, h_{B}=\lambda h_{A}$ and $d_{B}=\lambda d_{A}$ for some $\lambda>0$ ).

An example with $b_{A}=1, h_{A}=1, d_{A}=0$ and $\lambda=2$ is given in Fig. 4 (left), where the common components (two vertical lines at $\varphi=0$ and $\varphi= \pm \pi$ are indicated in thick gray. It is known [11] that there are four aspects in this case, and this can be checked on the figure: there are two connected components of the complement of the singular locus over each cylinder, one bounded and the other unbounded.

2. "Symmetric" manipulator where the moving triangle is the image of the base triangle by an indirect isometry of the plane (i.e. $b_{B}=b_{A}, h_{B}=-h_{A}$ and $d_{B}=d_{A}$ ).

An example with $b_{A}=b_{B}=2, h_{A}=-h_{B}=1$ and $d_{A}=d_{B}=0.5$ is given in Fig. 4 (right). Here the common component indicated in thick gray is described by $\varphi-2 \theta= \pm \pi$. It is known [15] that there are two aspects in this case.

We now turn to the cases when the first genericity assumption is fulfilled, but the second is not, that is, when the curve of zeros has components which are vertical lines $\varphi=$ constant. The existence of such values of $\varphi$ can be detected by computing 

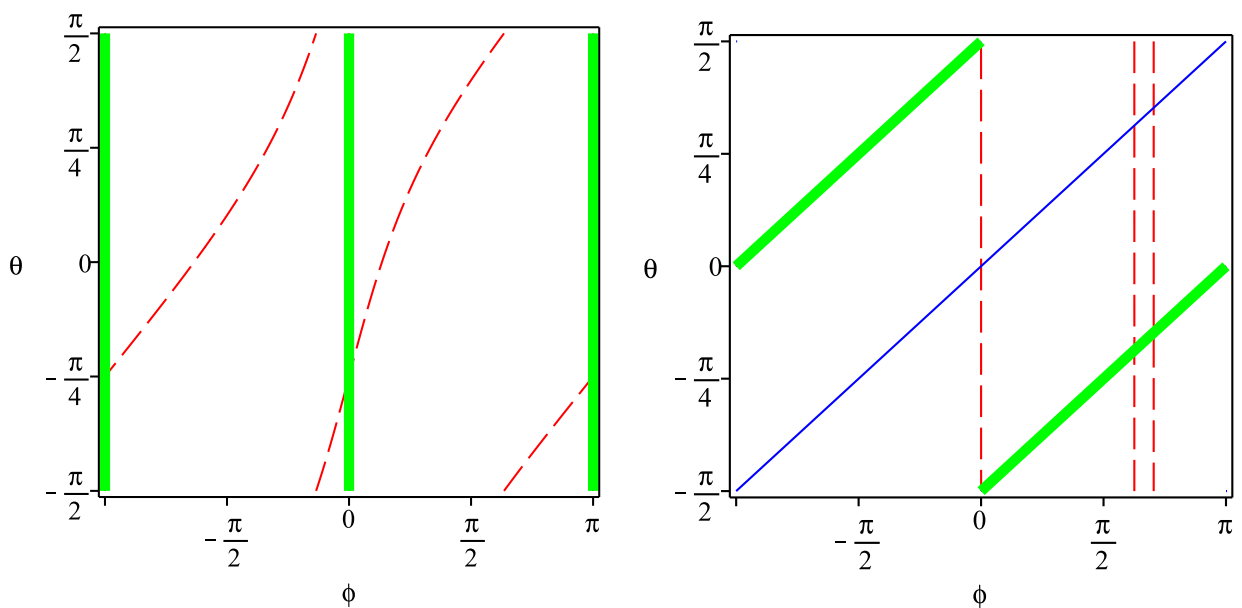

Fig. 4. Example of a "similar" manipulator (left) and of a "symmetric" manipulator (right)

the resultant of the coefficients of $\cos \theta$ and $\sin \theta$ in (2) with respect to $\tan (\varphi / 2)$. This resultant is a polynomial expression in $b_{A}, h_{A}, d_{A}, b_{B}, h_{B}, d_{B}$, whose factors which possibly vanish are the following

1. $d_{A} h_{B}-h_{A} d_{B}$, which vanishes when the angles $\alpha_{1}=\left(\overrightarrow{A_{1} A_{2}}, \overrightarrow{A_{1} A_{3}}\right)$ and $\beta_{1}=\left(\overrightarrow{B_{1} B_{2}}, \overrightarrow{B_{1} B_{3}}\right)$ are equal modulo $\pi$,

2. $b_{A}-b_{B}$, which vanishes when $A_{1} A_{2}=B_{1} B_{2}$,

3. $h_{A}^{2}+d_{A}^{2}-h_{B}^{2}-d_{B}^{2}$, which vanishes when $A_{1} A_{3}=B_{1} B_{3}$,

4. $b_{A}^{2} h_{A}^{2}\left(h_{B}^{2}+\left(b_{B}-d_{B}\right)^{2}\right)-b_{B}^{2} h_{B}^{2}\left(h_{A}^{2}+\left(b_{A}-d_{A}\right)^{2}\right)$, which vanishes when the height from vertex $A_{1}$ in the triangle $A_{1} A_{2} A_{3}$ is equal to the height from vertex $B_{1}$ in $B_{1} B_{2} B_{3}$.

The presence of vertical lines $\varphi=$ constant in the curve of zeros could forbid the gluing of a bounded component of the complement of the singular locus over one cylinder with the unbounded component over the other cylinder only in the case when two distinct vertical lines are contained in the same cylinder $T_{i}$. We sketch the argument showing that this cannot happen.

We may assume that the height from $A_{1}$ is not equal to the height from $B_{1}$, i.e. the factor 4 above does not vanish. Indeed, if the heights in the triangle $B_{1} B_{2} B_{3}$ are all three equal to the corresponding heights in the triangle $A_{1} A_{2} A_{3}$, then the triangles are equal or symmetric, which is excluded.

In case factor 1 vanishes, there are two vertical lines in the curve of zeros: the lines $\varphi=0$ and $\varphi=\pi$. These two lines cannot be contained in the same cylinder since one is obtained from the pther by the translation $\varphi \mapsto \varphi+\pi$.

We assume now that factors 1 and 4 do not vanish. If only one of the two factors 2 or 3 vanishes, then there is just one vertical line in the curve of zeros $\left(\varphi=0\right.$ or $\varphi=\alpha_{1}-\beta_{1}$, respectively). So we are left with the case where factors 2 and 3 both vanish, i.e $A_{1} A_{2}=B_{1} B_{2}$ and $A_{1} A_{3}=B_{1} B_{3}$. Moreover, we can assume that the heights from $B_{2}$ and from $B_{3}$ are equal respectively to the height from $A_{2}$ and $A_{3}$; otherwise, we could choose another vertex for $A_{1}$. We deduce that the parameters satisfy $b_{B}=b_{A}, h_{B}=h_{A}$ and $d_{B}=-d_{A}$. In this case there are two vertical lines in the curves of zeros, the lines $\varphi=0$ and $\varphi=\alpha_{1}-\beta_{1}$. These lines are tangent to the same branch of the curve of poles, at points with $\theta=0$ and $\theta=\alpha_{1}$ respectively. Hence, they are not contained in the same cylinder. An example of such a manipulator is given in Fig. 5.

This concludes the discussion of the non generic cases with the following

Theorem 2. The only non generic case when there are four aspects instead of two is when the base and moving triangles are similar (assuming neither is flat).

The case where one or the other of the triangles are flat has not been included in this discussion. It would need a special treatment, taking into account $h_{A}=0$ or $h_{B}=0$.

\subsection{Repartition of the solutions of the Direct Kinematic Problem (DKP)}

The fact that there are two aspects imply that the solutions of the DKP are equally distributed in each aspect, by a simple argument using topological degree of mappings. Recall that the topological degree of a smooth mapping $f: M \rightarrow N$ between compact connected oriented macannifolds of the same dimension can be computed at a point $q \in N$ which is not a critical value of $f$ as the sum of the signs $( \pm 1)$ of the Jacobian determinants of $f$ at all points $p \in f^{-1}(q)$ (i.e., one counts +1 if $f$ preserves the orientation at $p$ and -1 if it reverses the orientation. The degree is independant of the choice of the regular value $q$, see for instance [16]. 

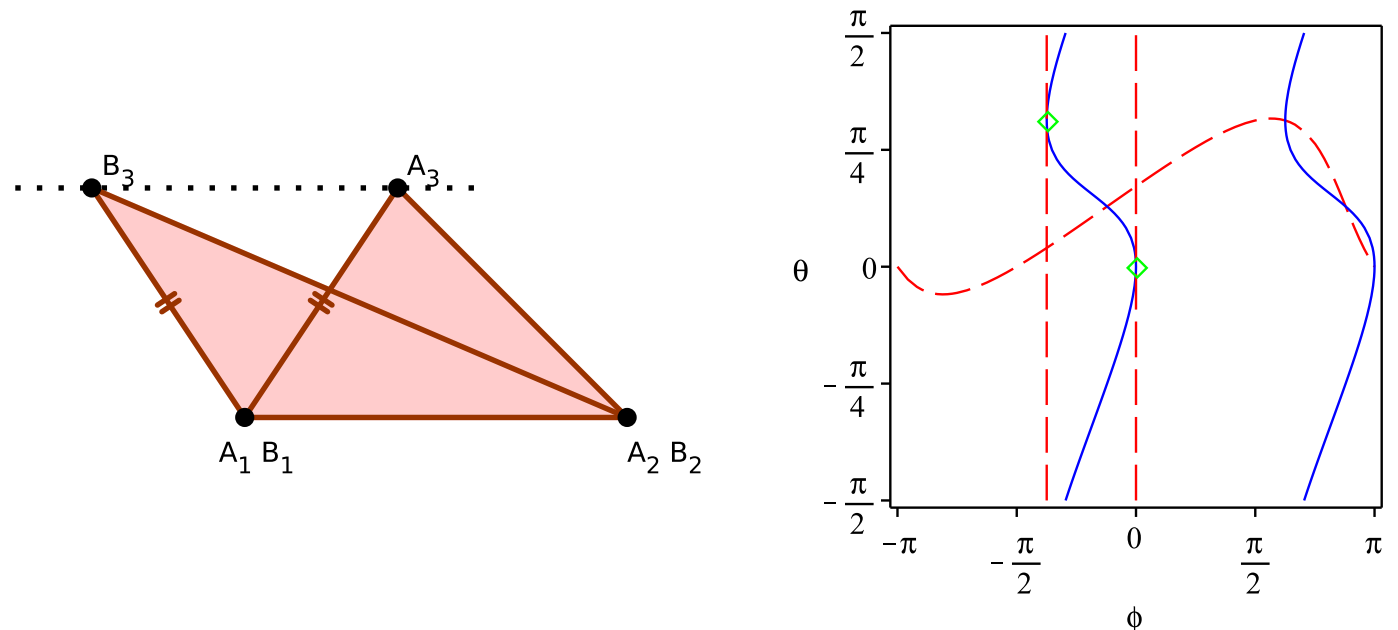

Fig. 5. Non generic manipulator with $b_{A}=b_{B}=5, h_{A}=h_{B}=3, d_{A}=-d_{B}=2$

We work here with the actual workspace $W$, which can be described as the product of the circle $S^{1}$ (with coordinate $\varphi$ ) with the plane $\mathbb{R}^{2}$ (with coordinates $(x, y)$ ). The Inverse Kinematic Mapping (IKM) is a mapping from the workspace $W \simeq S^{1} \times \mathbb{R}^{2}$ to the actuated joint space contained in $\mathbb{R}^{3}$. In each aspect, the sign of the Jacobian determinant of the IKM is constant. Hence, each of the two aspects is characterized by the sign of the Jacobian determinant.

The IKM can be continuously extended to a mapping from the product of $S^{1}$ with the 2-sphere $S^{2}$ (compactification of $\mathbb{R}^{2}$ ) to the 3 -sphere $S^{3}$ (compactification of $\mathbb{R}^{3}$ ). Indeed, when the vertex $B_{1}$ with coordinates $(x, y)$ goes to infinity, the lengths of all three legs $A_{i} B_{i}$ tend to infinity. The extended continuous mapping between compact connected oriented manifolds $S^{1} \times S^{2} \rightarrow S^{3}$ is not surjective, hence its topological degree is 0 . It follows that, if $q \in \mathbb{R}^{3}$ is a regular value of IKM, the sum of the signs of the Jacobian determinants at all solutions of the DKP for $q$ is zero. Hence, there are as many solutions of the DKP for which it is positive as there are for which it is negative. This proves

Theorem 3. For a non similar 3-RPR manipulator, the solutions of the DKP for a regular value of the IKM are equally distributed in each aspect.

\section{Conclusion}

The coordinates $\left(\varphi, \theta, r_{1}\right)$ used for the modified workspace enabled us to express $r_{1}$ as a function of the angular coordinates $\varphi$ and $\theta$ on the singular surface. This description of the singular surface was then used in simple topological arguments to prove that, for a generic 3-RPR manipulator, the complement of the singular locus in the workspace has two connected components, i.e. the manipulator has two aspects. We showed how to design a singularity-free path between any two points in the same aspect. We also established that the only non generic case when there are four aspects instead of two is when the moving triangle is directly similar to the base triangle (assuming neither triangle is flat). We gave a proof that the solutions of the DKP are equally distributed in the two aspects.

\section{Acknowledgments}

The author thanks Philippe Wenger for his pertinent advice and the referees for their useful comments. He has been supported for this research by ANR project SIROPA.

\section{References}

[1] C. Innocenti and V. Parenti-CAstelli, "Singularity-free Evolution From One Configuration to Another in Serial and Fully-Parallel Manipulators", Proc. ASME Design Technical Conferences, DE-Vol. 45, Spatial mechanisms and mechanical systems, pp 553-560, 1992.

[2] P. Wenger and D. Chablat, "Workspace and assembly-modes in fully-parallel manipulators: a descriptive study", Advances in Robot Kinematics and Computational Geometry, Kluwer Academic Publishers, pp. 117-126, 1998.

[3] P.R. MCAREE and R.W. DANIEL, "An explanation of never-special assembly changing motions for 3-3 parallel manipulators", The International Journal of Robotics Research, 18(6): 556-574, 1999. 
[4] H. Bamberger, A. Wolf and M. Shoham, "Assembly mode changing in parallel mechanisms", IEEE Transactions on Robotics, 24(4): 765-772, 2008.

[5] A. Hernandez, O. Altuzarra, V. Petuya and E. Macho, "Defining conditions for nonsingular transitions between assembly modes", IEEE Transactions on Robotics, 25(6): 1438-1447, 2009.

[6] M.L. Husty, "Non-singular assembly mode change in 3-RPR-parallel manipulators". In Computational Kinematics: Proceedings of the 5th International Workshop on Computational Kinematics, pp 51-60. Springer Verlag, 2009.

[7] K.H. HunT, "Structural kinematics of in-parallel actuated robot arms", J. of Mechanisms, Trans. and Aut. in Design 105(4): 705-712, 1983.

[8] G.R. PENNOCK and D.J. KASSNER, "Kinematic analysis of a planar eight-bar linkage: application to a platform-type robot", ASME Proc. of the 21th Biennial Mechanisms Conf., pp37-43, Chicago, September 1990.

[9] C.M. Gosselin and J-P. Merlet, "On the direct kinematics of planar parallel manipulators: special architectures and number of solutions”, Mechanism and Machine Theory, 29(8): 1083-1097, 1994.

[10] J. SEFRIOUI and C.M. GossELIN, "On the quadratic nature of the singularity curves of planar three-degree-of-freedom parallel manipulators", Mechanism and Machine Theory, 30(4): 533-551, 1995.

[11] X. Kong and C.M. Gosselin, "Determination of the uniqueness domains of 3-RPR planar parallel manipulators with similar platforms", Proc. of the 2000 ASME Design Engineering Technical conferences and Computers and Information in Engineering Conference, Baltimore, Sept 10-13, 2000.

[12] I. Bonev, D. Zlatanov, and C. Gosselin, "Singularity Analysis of 3-DOF Planar Parallel Mechanisms via Screw Theory”, ASME Journal of Mechanical Design, 125(3): 573-581, 2003.

[13] P. Wenger, D. Chablat. and M. Zein, "Degeneracy study of the forward kinematics of planar 3-RPR parallel manipulators", ASME Journal of Mechanical Design, 129(12): 1265-1268, 2007.

[14] J-P. MERLET, Parallel Robots, Springer 2006.

[15] P. WEnger and D. CHABLAT, "Kinematic analysis of a class of analytic planar 3-RPR parallel manipulators". In Computational Kinematics: Proceedings of the 5th International Workshop on Computational Kinematics, pp 43-50. Springer Verlag, 2009.

[16] J.MiLnOR, Topology from the Differentiable Viewpoint, The University Press of Virginia 1969. 